\title{
A Paridade do Poder de Compra: Existe um Quebra-Cabeça?
}

\author{
- Fernando de Holanda Barbosa*
}

\section{RESUMO}

Este trabalho mostra que o quebra-cabeça da paridade do poder de compra é um artefato estatístico produzido pelo fato de que a taxa de câmbio real de equilíbrio de longo prazo não é constante, mas varia ao longo do tempo. Este fato implica que o coeficiente de inércia é viciado para cima.

\section{Palavras-Chave}

paridade do poder de compra, taxa de câmbio real, taxa de câmbio real de equilíbrio de longo prazo

\section{ABSTRACT}

This paper shows that the purchasing power parity puzzle is a statistical artifact produced by the fact that the long run equilibrium real exchange rate is not constant, but changes throughout time. This fact implies that the inertia coefficient has an upward bias.

\section{KEYWORDS}

purchasing power parity, real exchange rate, long-run equilibrium real exchange rate

\section{JEL CLASSIFICATION}

F3I, F32, F4I

* Professor da Escola de Pós-Graduação de Economia da Fundação Getúlio Vargas. Endereço para contato: Fundação Getúlio Vargas - Praia de Botafogo, 190, sala I 100 - Rio de Janeiro - RJ. CEP: 22253-900. E-mail: fholanda@fgv.br. (Recebido em novembro de 2005. Aceito para publicação em julho de 2007). 


\section{INTRODUÇÃO}

Uma grande quantidade de trabalhos empíricos tem testado a hipótese da paridade de poder de compra, que supóe a taxa de câmbio real constante no longo prazo. ${ }^{1} \mathrm{~A}$ conclusão destes trabalhos resultou no conhecido "quebra-cabeça" da paridade do poder de compra, que é produzido pelas estimativas obtidas da seguinte regressão: ${ }^{2}$

$$
q_{t}=\mu(1-\rho)+\rho q_{t-1}+\varepsilon_{t}
$$

A letra $q$ representa o logaritmo da taxa de câmbio real $Q$ definida por

$$
Q=\frac{S P^{*}}{P}
$$

$S$ é a taxa de câmbio nominal, $P^{*}$ o índice de preços externo, $P$ o índice de preços doméstico, $\mu$ a taxa de câmbio real de equilíbrio de longo prazo, $\rho$ o coeficiente de inércia e $\varepsilon$ o termo estocástico.

O "quebra-cabeça" da paridade do poder de compra é baseado no fato de que as estimativas do parâmetro $\rho$ ou não rejeitam a hipótese de raiz unitária, ou quando rejeitam, as estimativas deste parâmetro estão no intervalo compreendido entre 0,8 e 0,9 , que implicam uma meia vida do processo entre três e quatro anos. ${ }^{3}$ Como explicar, então, que o processo de ajuste da taxa de câmbio real seja tão lento?

Este trabalho tem como objetivo mostrar que o "quebra-cabeça" é na verdade um artefato estatístico, produzido pelo fato de que a taxa de câmbio real de equilíbrio de longo prazo não é constante, mas varia ao longo do tempo. A abordagem que será usada para demonstrar este fato concilia a pesquisa acadêmica preocupada em testar a hipótese da paridade de poder de compra com a pesquisa orientada para a política econômica que procura estimar a taxa de câmbio real de equilíbrio de longo

l A paridade do poder de compra tem uma longa história na teoria econômica e certamente uma das referências clássicas é Cassel (1918). A justificativa teórica para a paridade do poder de compra seria a lei do preço único dos bens transacionados no comércio internacional. Todavia, Harrod (1939), Balassa (1964) e Samuelson (1964) chamaram atenção para o fato de que existem bens que não são comercializados internacionalmente. Seus preços diferem entre os países, pois não é possível um processo de arbitragem entre os mesmos. Este fato implica que a taxa de câmbio real de equilíbrio de longo prazo não é constante, pois depende do progresso tecnológico na economia, como será visto na seção 2 .

2 O quebra-cabeça da paridade do poder de compra foi formulado por Rogoff (1996), e existem na literatura várias tentativas de resolvê-lo. Uma das mais recentes consiste em supor que o processo de reversão para a média não é linear. Veja, por exemplo, Michael, Nobay e Peel (1997) e Taylor, Peel e Sarno (2001).

3 O tempo que um choque leva para que metade do mesmo seja dissipado é denominado meia vida do processo. Uma resenha dos vários procedimentos estatísticos empregados na estimativa da regressão (1) pode ser encontrada em Rogoff (1996). 
prazo. ${ }^{4}$ Uma consequência desta abordagem é que ela não implica que a não rejeição da hipótese de raiz unitária para a taxa de câmbio real seja equivalente à rejeição da hipótese de paridade do poder de compra, pois a taxa de câmbio real pode ser uma variável não estacionária e cointegrada com a taxa de câmbio real de longo prazo. ${ }^{5}$

O trabalho está organizado do seguinte modo: a Seção 2 apresenta uma resenha sucinta de alguns modelos largamente usados na literatura de macroeconomia aberta que especificam as variáveis que afetam a taxa de câmbio real de equilíbrio de longo prazo; a Seção 3 calcula o viés do estimador de mínimos quadrados ordinário da regressão (1) quando a taxa de câmbio real de equilíbrio de longo prazo não é constante; a Seção 4 analisa várias hipóteses quanto ao processo estocástico da taxa de câmbio real de equilíbrio de longo prazo e deduz o processo correspondente à taxa de câmbio real de curto prazo; a Seção 5 conclui o trabalho.

\section{A TAXA DE CAMMBIO REAL DE EQUILÍBRIO DE LONGO PRAZO É CONS- TANTE?}

Os modelos de Mundell-Fleming, da economia dependente e de otimização intertemporal, sugerem algumas variáveis que afetam a taxa de câmbio real de equilíbrio de longo prazo. Esta seção apresenta uma resenha sucinta destes modelos com a preocupação de explicitar as respectivas equações que determinam a taxa de câmbio real de equilíbrio de longo prazo. No curto prazo, choques nominais afetam a taxa de câmbio real, como no conhecido modelo de ultrapassagem (overshooting) de Dornbusch (1976), mas a taxa de câmbio real reverte para o valor de equilíbrio de longo prazo com o passar do tempo, ou seja, a dicotomia clássica entre variáveis nominais e reais aplica-se neste caso. ${ }^{6}$

\subsection{Modelo Mundell-Fleming}

Na curva IS do modelo Mundell-Fleming (MUNDELL, 1963; FLEMING, 1962) a poupança depende da renda disponível $(y-g+f$, onde $y$ é o produto real, $g$ os gastos do governo, e $f$ o déficit público), o investimento $(I)$ é função da taxa de juros real

4 Para trabalhos orientados para a política econômica, ver, por exemplo, os artigos na coletânea organizada por Hinkle e Montiel (1999). Ver, também, Edwards (1989) e Williamson (1974).

$5 \mathrm{Na}$ apresentação didática da hipótese da paridade do poder de compra é usual fazer-se a distinção entre a paridade absoluta $(Q=1)$ e a paridade relativa $(Q=$ constante $)$. Nesta formulação estamos generalizando a hipótese da paridade do poder de compra para abranger também o caso em que a taxa de câmbio real converge para a taxa de câmbio real de equilíbrio de longo prazo, que pode ser ou não estacionária.

6 No modelo de Obstfeld e Rogoff (1995) esta dicotomia não é válida. 
$(r)$, e o déficit em conta corrente do balanço de pagamentos depende da taxa de câmbio real. Isto é:

$$
S(y-g+f)=I(r)+f+c(q)
$$

Numa economia aberta pequena a arbitragem da taxa de juros implica que a diferença entre as taxas de juros reais, doméstica e externa, é igual à variação da taxa de câmbio real:

$$
r=r^{*}+\dot{q}
$$

No longo prazo, quando a economia estiver em pleno emprego, o produto real é igual ao produto potencial e a taxa de câmbio real é igual à taxa de equilíbrio de longo prazo. Isto é: $y=\bar{y}, \dot{q}=0$, e $q=\bar{q}$. Logo, a taxa de câmbio real de equilíbrio de longo prazo depende do déficit público, dos gastos do governo, da taxa de juros real internacional e do produto real potencial da economia, com as derivadas parciais indicando como a taxa de câmbio real de equilíbrio de longo prazo reage quando estas variáveis mudam. Isto é: ${ }^{7}$

$$
\bar{q}=\bar{q}\left(f, g, r^{*}, \bar{y}\right), \frac{\partial \bar{q}}{\partial f}<0, \frac{\partial \bar{q}}{\partial g}<0, \frac{\partial \bar{q}}{\partial r^{*}}>0, \frac{\partial \bar{q}}{\partial \bar{y}}>0
$$

A taxa de câmbio real de equilíbrio de longo prazo aumenta quando: i) o déficit público diminui; ii) o gasto do governo diminui; iii) a taxa real de juros externa aumenta e iv) o produto real potencial da economia aumenta.

\subsection{Modelo da Economia Dependente}

O modelo da economia dependente supõe uma economia com dois setores, um produzindo bens comercializáveis e o outro setor bens não comercializáveis. ${ }^{8} \mathrm{~A}$ taxa de câmbio real é calculada através da seguinte fórmula:

7 No longo prazo, a equação (3) é dada por: $S(\bar{y}-g+f)=I\left(r^{*}\right)+f+c(\bar{q})$. Os sinais das derivadas parciais podem ser facilmente deduzidos a partir desta expressão. Por exemplo, quando o déficit público aumenta, a poupança doméstica aumenta, mas não o suficiente para financiá-lo. Logo, a taxa de câmbio real tem que diminuir para que a poupança externa financie parte do déficit público.

8 O modelo da economia dependente, desenvolvido por Salter (1959) e Swan (1960), admite um país pequeno que não afeta os preços dos bens exportáveis e importáveis, com a relação de trocas exógena ao modelo. A versão apresentada aqui é devida a Obstfeld e Rogoff (1996). 


$$
Q=\frac{S P^{*}}{P}=\frac{S P_{T}^{*(1-\alpha)} P_{N}^{* \alpha}}{P_{T}^{1-\alpha} P_{N}^{\alpha}}=\left(\frac{p^{*}}{p}\right)^{\alpha}
$$

onde cada índice de preços é uma média geométrica dos preços de cada um dos setores, e o peso de cada setor é o mesmo nos dois países. O comércio internacional iguala os preços dos bens comercializáveis, $S P_{T}^{*}=P_{T}$. A taxa de câmbio real depende, portanto, da razão entre os dois preços relativos, $p^{*}=P_{N}^{*} / P_{T}^{*}$ e $p=P_{N} / P_{T}$.

A função de produção de cada setor tem retornos constantes de escala e o progresso tecnológico é neutro:

$$
\begin{aligned}
& Y_{T}=A_{T} F\left(K_{T}, L_{T}\right) \\
& Y_{N}=A_{N} G\left(K_{N}, L_{N}\right)
\end{aligned}
$$

onde $Y$ representa o produto real, $K$ o estoque de capital, $L$ a quantidade de mão de obra, e $A$ o progresso tecnológico, e o índice de cada variável indica o setor da economia.

A quantidade de mão de obra total disponível na economia é fixa:

$$
L_{T}+L_{N}=L
$$

e existe mobilidade da mão de obra entre os dois setores.

A hipótese de país pequeno mais mobilidade de capital significa dizer que a taxa de retorno do capital no país é igual à taxa de juros externa:

$$
r=r^{*}
$$

As empresas de cada um dos setores são competitivas e os valores das produtividades marginais dos fatores são iguais aos respectivos preços:

$$
\begin{aligned}
& A_{T} f^{\prime}\left(k_{T}\right)=r^{*} \\
& A_{T}\left[f\left(k_{T}\right)-k_{T} f^{\prime}\left(k_{T}\right)\right]=\omega \\
& p A_{N} g^{\prime}\left(k_{N}\right)=r^{*} \\
& p A_{N}\left[g\left(k_{N}\right)-k_{N} g^{\prime}\left(k_{N}\right)\right]=\omega
\end{aligned}
$$


O preço dos comercializáveis serviu como unidade de conta $\left(P_{T}=1\right)$, $\omega$ é o salário real em termos de bens comercializáveis, e as funções de produção foram escritas em função das respectivas relações capital/mão de obra: $y_{T}=f\left(k_{T}\right), y_{N}=g\left(k_{N}\right)$ onde $y_{T}=Y_{T} / L_{T}, k_{T}=K_{T} / L_{T}, y_{N}=Y_{N} / L_{N}$, e $k_{N}=K_{N} / L_{N}$.

O sistema de equações formado pelas quatro equações anteriores tem quatro incógnitas, as duas relações capital/mão de obra, o salário real e o preço relativo dos bens não comercializáveis, e três variáveis exógenas, as duas variáveis de progresso tecnológico e a taxa de juros internacional. Em princípio, portanto, é possível escrever cada uma das variáveis endógenas como função das três variáveis exógenas. Logo, o preço relativo dos bens não comercializáveis depende do comportamento destas três variáveis. Isto é: ${ }^{9}$

$$
\bar{p}=\bar{p}\left(A_{T}, A_{N}, r^{*}\right), \frac{\partial \bar{p}}{\partial A_{T}}>0, \frac{\partial \bar{p}}{\partial A_{N}}<0, \frac{\partial \bar{p}}{\partial r^{*}}<0
$$

Os sinais das derivadas parciais mostram a resposta do preço relativo a mudanças em cada uma das variáveis exógenas do modelo: i) aumento da produtividade na produção dos bens comercializáveis aumenta o preço relativo dos bens não comercializáveis; ii) aumento da produtividade dos bens não comercializáveis diminui o preço relativo dos bens não comercializáveis e iii) aumento da taxa de juros internacional diminui o preço relativo dos bens não comercializáveis.

Neste modelo de uma economia dependente a curva de demanda do bem não comercializável não afeta o preço, mas apenas a quantidade produzida, como mostra a Figura 1. Todavia, se o custo marginal de produção não for constante a curva de demanda afeta o preço, como será mostrado a seguir.

A despesa do consumidor na compra dos dois bens obedece a seguinte restrição orçamentária:

$$
P_{N} C_{N}+P_{T} C_{T}=P C
$$

onde $P$ e $C$ são os índices de preços e quantidades dos dois bens. Supondo-se que a função utilidade do consumidor seja Cobb-Douglas, estes índices são dados por

$$
P=P_{T}^{1-\alpha} P_{N}^{\alpha}=P_{T}\left(P_{N} / P_{T}\right)^{\alpha}=P_{T} p^{\alpha}
$$

9 O exercício de estática comparativa com o sistema de equações (11)-(14) permite obter os sinais das derivadas parciais. Todavia, os resultados são intuitivos. Por exemplo, o aumento do progresso tecnológico na produção de bens comercializáveis reduzirá o seu custo marginal, e fará com que o preço relativo dos bens não comercializáveis aumente. 


$$
C=C_{T}^{1-\alpha} C_{N}^{\alpha}
$$

onde $\alpha$ é a proporção da despesa com a aquisição do bem não comercializável,

$$
P_{N} C_{N}=\alpha P C
$$

e 1- $\alpha$ é a proporção da despesa na compra do bem comercializável:

$$
P_{T} C_{T}=(1-\alpha) P C
$$

FIGURA 1

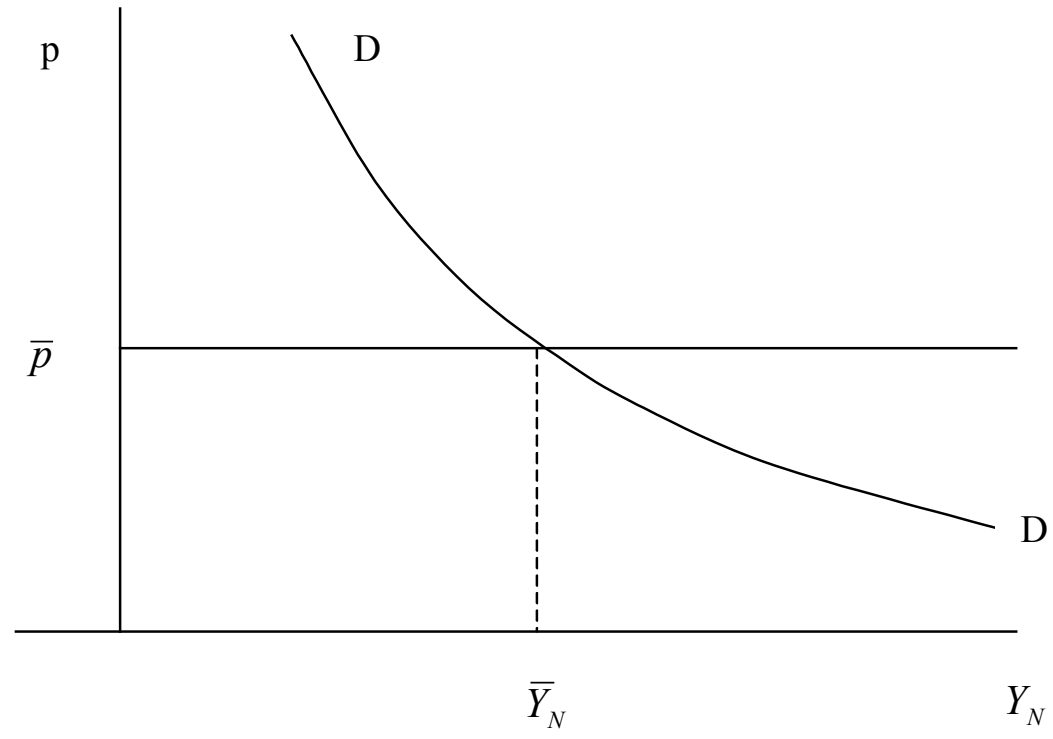

As equações de demanda dos dois bens são, então, dadas pelas expressões:

$$
\begin{aligned}
& C_{N}=\alpha p^{-(1-\alpha)} C \\
& C_{T}=(1-\alpha) p^{\alpha} C
\end{aligned}
$$

As equações de oferta dos dois bens supõem que os custos marginais de produção são crescentes. Isto é: 


$$
\begin{gathered}
Y_{N}=Y_{N}(p), \frac{\partial Y_{N}}{\partial p}>0 \\
Y_{T}=Y_{T}(p), \frac{\partial Y_{T}}{\partial p}<0
\end{gathered}
$$

O equilíbrio no mercado dos bens não comercializáveis ocorre quando a oferta for igual à demanda:

$$
Y_{N}=C_{N}+g_{N}
$$

onde $g_{N}$ é a compra de bens não comercializáveis pelo governo.

O saldo em conta corrente do balanço de pagamento $(\dot{b})$ é igual à soma dos juros recebidos $(r b)$ com o excesso de produção dos bens comercializáveis sobre o total do consumo interno do mesmo, seja privado ou público $\left(g_{T}\right)$. Isto é:

$$
\dot{b}=r b+Y_{T}-C_{T}-g_{T}
$$

Quando a conta corrente estiver em equilíbrio $(\dot{b}=0)$ a equação (26) transformase em:

$$
Y_{T}=C_{T}+g_{T}-r b
$$

O sistema formado pelas equações (25) e (26), de equilíbrio no mercado dos bens não comercializáveis, está representado graficamente na Figura 2. Nesta figura a curva IE mostra a combinação do preço relativo dos bens não comercializáveis e do consumo que corresponde ao equilíbrio interno, enquanto a curva EE mostra a combinação destas variáveis que produz o equilíbrio externo da economia. Mudanças destas curvas alteram o equilíbrio de longo prazo da economia. 
FIGURA 2

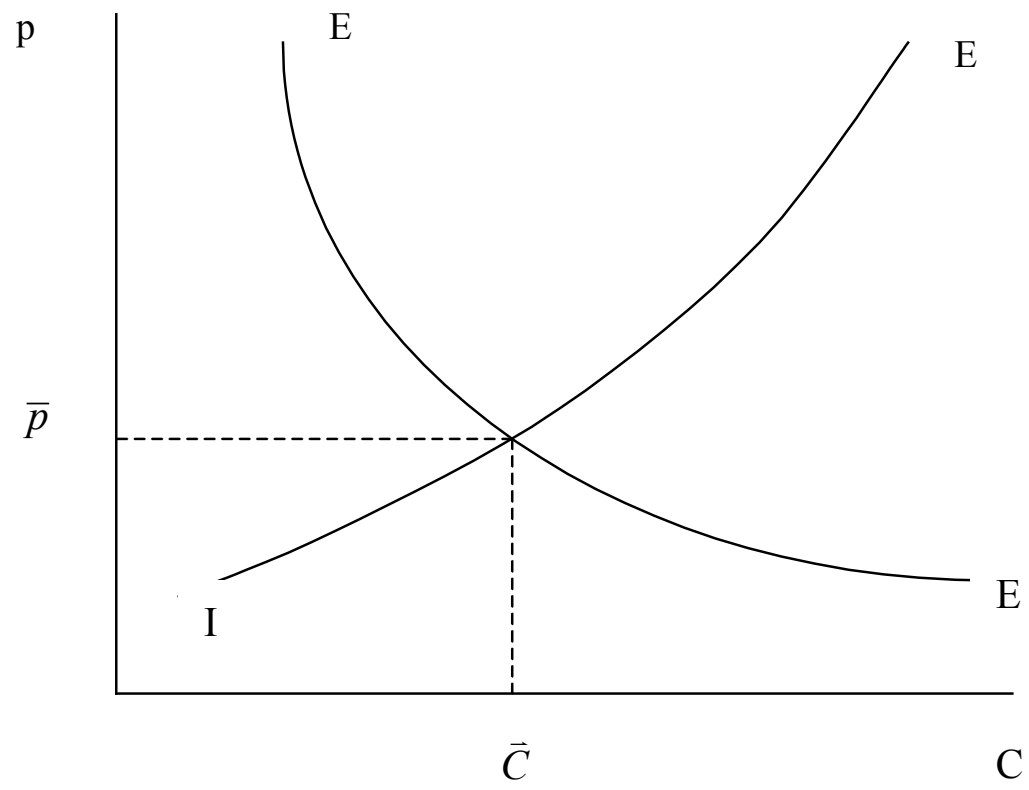

O preço relativo dos bens não comercializáveis aumenta quando os gastos do governo com bens não comercializáveis aumentam, e diminui quando os gastos do governo com bens comercializáveis aumentam. Os sinais das derivadas parciais da função do preço dos bens não comercializáveis com relação à taxa de juros internacional e ao estoque de ativos externos dependem da situação do país, se credor ou devedor. Quando o país é credor, os sinais destas duas derivadas parciais são positivos; caso contrário, elas são negativas. Este modelo permite, portanto, escrever o preço relativo dos bens não comercializáveis de equilíbrio de longo prazo como função dos gastos do governo na compra dos dois bens, da taxa de juros internacional e do estoque de ativos externos:

$$
\bar{p}=\bar{p}\left(g_{N}, g_{T}, r, b\right), \frac{\partial \bar{p}}{\partial g_{N}}>0, \frac{\partial \bar{p}}{\partial g_{T}}<0, \frac{\partial \bar{p}}{\partial r} \geq 0, \frac{\partial \bar{p}}{\partial b} \geq 0
$$

\subsection{Modelo de Otimização Intertemporal}

No modelo de otimização intertemporal, o agente representativo maximiza o valor presente do fluxo de utilidade, 


$$
\int_{0}^{\infty} e^{-\delta t} \frac{C^{1-\sigma}}{1-\sigma} d t
$$

onde $\delta$ é a taxa de preferência intertemporal e o inverso do parâmetro $\sigma$ é a elasticidade de substituição do consumo. A restrição orçamentária do agente é dada por:

$$
\dot{b}=C_{T}+p C_{N}+\tau+\left(i-\pi_{T}^{*}\right) b-Y_{T}-p Y_{N}
$$

onde $b$ é o estoque da dívida medida em termos dos bens comercializáveis, $\tau$ é um imposto sem efeito na alocação de recursos (do tipo lump sum), $i$ é a taxa de juros externa paga pelo país, $\pi_{T}^{*}$ é a taxa de inflação externa dos bens comercializáveis, e os demais símbolos têm o mesmo significado dos modelos anteriores.

\section{FIGURA 3}

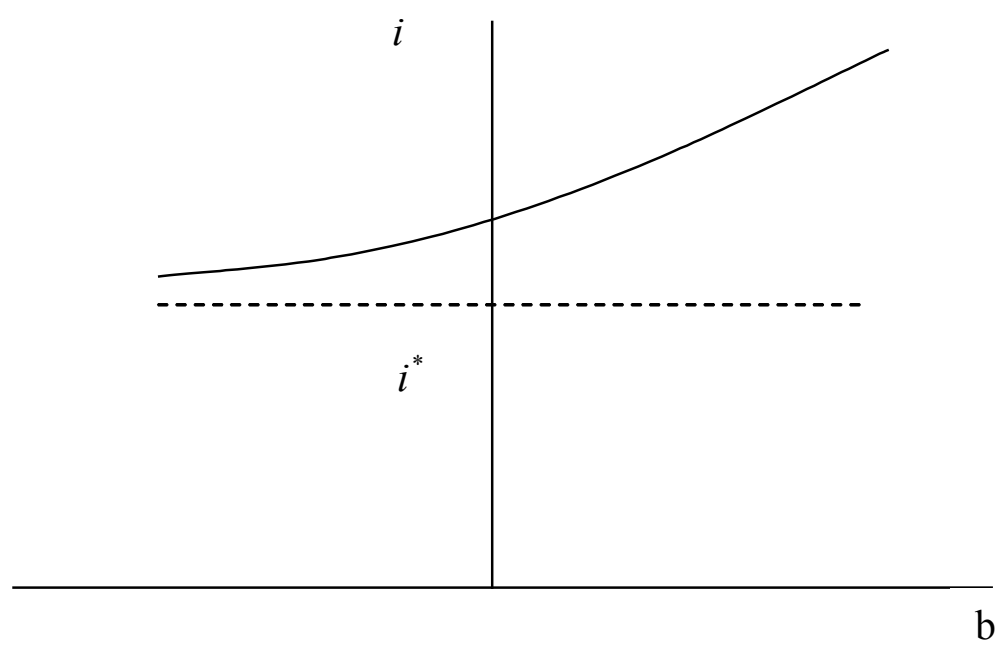

A taxa de juros externa do país tem um prêmio de risco que depende do estoque da sua dívida externa, de acordo com:

$$
i=i^{*}+\theta(b), \quad \theta^{\prime}(b) \geq 0, \theta(0)>0
$$


onde $i^{*}$ é a taxa de juros internacional e o prêmio de risco $\theta(b)$ é uma função não decrescente do estoque da dívida com o formato da Figura 3.

Levando-se em conta que $C_{T}+p C_{N}=p^{\alpha} C$ e a equação anterior do prêmio de risco, a restrição orçamentária pode ser escrita como: ${ }^{10}$

$$
\dot{b}=p^{\alpha} C+\tau+\left(r^{*}+\theta(b)\right) b-Y
$$

onde $Y=Y_{T}+p Y_{N}$ é o produto real da economia medido em termos dos bens comercializáveis, e $r^{*}$ é a taxa de juros real externa.

O hamiltoniano de valor corrente para resolver o problema de otimização do agente é dado por:

$$
H=\frac{C^{1-\sigma}}{1-\sigma}+\lambda\left(p^{\alpha} C+\tau+\left(r^{*}+\theta(b)\right) b-Y\right)
$$

onde $\lambda$ é a variável de coestado. As condições de primeira ordem deste problema são:

$$
\frac{\partial H}{\partial C}=C^{-\sigma}+\lambda p^{\alpha}=0
$$

$\mathrm{e}$

$$
\dot{\lambda}=\lambda \delta-\frac{\partial H}{\partial b}=\lambda \delta-\lambda\left(r^{*}+b \theta^{\prime}(b)\right)
$$

A solução ótima tem que satisfazer à condição de transversalidade:

$$
\lim _{t \rightarrow \infty} \lambda b e^{-\delta t}=0
$$

O mercado de bens não comercializáveis está em equilíbrio quando a oferta for igual à demanda. Isto é:

$$
Y_{N}=\alpha p^{-(1-\alpha)} C+g_{N}
$$

10 A equação (30) permite escrever: $\dot{b}=p^{\alpha} C+\tau+\left(i^{*}-\pi_{T}^{*}+\theta(b)\right) b-Y$. Como $i^{*}-\pi_{T}^{*}=r^{*}$, obtém-se, então, a equação (32). 
Admite-se, como no modelo anterior, que as produções dos dois bens tenham custos marginais crescentes.

O governo financia-se integralmente através de imposto. Logo,

$$
\tau=g_{T}+p g_{N}
$$

O modelo formado pelas equações (32), (34), (35), (36) e (37) pode ser resolvido do seguinte modo: das equações (34) e (37) obtém-se C e $p$ em função de $\lambda$; substituem-se estes valores nas equações (32) e (35) para se obter um sistema dinâmico nas variáveis $b$ e $\lambda$. Neste sistema dinâmico calculam-se os elementos da matriz jacobiana e daí as condições para a trajetória de sela.

A taxa de câmbio real de equilíbrio de longo prazo neste modelo será definida para o estado estacionário do modelo, quando $\dot{\lambda}=\dot{b}=0$. Logo, o preço relativo dos bens não comercializáveis em equilíbrio de longo prazo depende da taxa de preferência intertemporal, dos gastos do governo e da taxa de juros internacional. Isto é:

$$
\bar{p}=\bar{p}\left(\delta, g_{N}, g_{T}, r^{*}\right), \frac{\partial \bar{p}}{\partial \delta}<0, \frac{\partial \bar{p}}{\partial g_{N}}>0, \frac{\partial \bar{p}}{\partial g_{T}}<0, \frac{\partial \bar{p}}{\partial r^{*}}>0
$$

Os sinais das derivadas parciais desta expressão mostram que o preço relativo dos bens não comercializáveis aumenta quando: i) a taxa de preferência intertemporal diminui; ii) os gastos do governo com bens não comercializáveis aumentam; iii) os gastos do governo com bens não comercializáveis diminuem, e iv) a taxa de juros real internacional aumenta.

\section{OQUEBRA-CABEÇA DA INÉRCIA}

O estimador de mínimos quadrados ordinário do parâmetro $\rho$ da regressão (l) é dado por:

$$
\hat{\rho}=\frac{\sum_{t=2}^{T}\left(q_{t-1}-\bar{q}\right) q_{t}}{\sum_{t=2}^{T}\left(q_{t-1}-\bar{q}\right)^{2}}
$$

Admita-se que a taxa de câmbio real de equilíbrio de longo prazo $\mu$ não seja constante, como supóe a hipótese de paridade do poder de compra, e que a diferença entre 
a taxa de câmbio real observada e a de equilíbrio de longo prazo siga um processo autorregressivo de primeira ordem. Isto é:

$$
q_{t}-\mu_{t}=\rho\left(q_{t-1}-\mu_{t-1}\right)+\varepsilon_{t}
$$

Esta equação também pode ser escrita como:

$$
q_{t}=\mu_{t}-\rho \mu_{t-1}+\rho q_{t-1}+\varepsilon_{t}
$$

Quando $\mu_{t}=\mu_{t-1}=\mu$, obtém-se a equação (1) usada para estimar a inércia da taxa de câmbio real. Todavia, se a taxa de câmbio real de equilíbrio de longo prazo é variável, de acordo com a equação anterior, o limite em probabilidade do estimador de mínimos quadrados ordinário do parâmetro $\rho$ é dado por:

$$
p \lim \hat{\rho}=\rho+p \lim \frac{\sum\left(q_{t-1}-\bar{q}\right)\left(\mu_{t}-\rho \mu_{t-1}\right)}{\sum\left(q_{t-1}-\bar{q}\right)^{2}}
$$

Quando $T \rightarrow \infty$ este limite em probabilidade é igual a:

$$
p \lim \hat{\rho}=\rho+\frac{\operatorname{cov}\left(q_{t-1}, \mu_{t}\right)-\rho \operatorname{cov}\left(q_{t-1}, \mu_{t-1}\right)}{\operatorname{var}(q)}
$$

Este limite pode ser reescrito como:

$$
p \lim \hat{\rho}=\rho+\frac{\operatorname{cov}\left(q_{t-1}, \mu_{t-1}\right)}{\operatorname{var}(q)}\left[\frac{\operatorname{cov}\left(q_{t-1}, \mu_{t}\right)}{\operatorname{cov}\left(q_{t-1}, \mu_{t-1}\right)}-\rho\right]
$$

O processo autorregressivo do desvio da taxa de câmbio real observada em relação à taxa de equilíbrio de longo prazo pode ser escrito usando-se o operador de defasagem $L$ do seguinte modo:

$$
(1-\rho L) q_{t}=(1-\rho L) \mu_{t}+\varepsilon_{t}
$$

ou ainda:

$$
q_{t}=\mu_{t}+\frac{\varepsilon_{t}}{1-\rho L}
$$

É fácil concluir desta expressão que: 
$\operatorname{cov}\left(q_{t-1}, \mu_{t-1}\right)=E\left(q_{t-1}-E q_{t-1}\right) \mu_{t-1}=E\left(\mu_{t-1}-E \mu_{t-1}\right) \mu_{t-1}=\operatorname{var}(\mu)$

e:

$$
\operatorname{cov}\left(q_{t-1}, \mu_{t}\right)=E\left(q_{t-1}-E q_{t-1}\right) \mu_{t}=E\left(\mu_{t-1}-E \mu_{t-1}\right) \mu_{t}=\operatorname{cov}\left(\mu_{t}, \mu_{t-1}\right)
$$

Logo, o limite em probabilidade do estimador de mínimos quadrados ordinário de $\rho$ é igual a:

$$
p \lim \hat{\rho}=\rho+\frac{\operatorname{var}(\mu)}{\operatorname{var}(q)}\left[\frac{\operatorname{cov}\left(\mu_{t}, \mu_{t-1}\right)}{\operatorname{var}(\mu)}-\rho\right]
$$

A razão entre a covariância de $\mu_{t}$ e $\mu_{t-1}$ e a variância de $\mu$ é o coeficiente de correlação entre estas duas variáveis: $\varphi=\operatorname{corr}\left(\mu_{t}, \mu_{t-1}\right)=\operatorname{cov}\left(\mu_{t}, \mu_{t-1}\right) / \operatorname{var} \mu$. Portanto, a expressão anterior pode ser escrita como:

$$
p \lim \hat{\rho}=\rho+\frac{\operatorname{var}(\mu)}{\operatorname{var}(q)}[\varphi-\rho]
$$

ou como a média ponderada dos dois coeficientes de correlação:

$$
p \lim \widehat{\rho}=\left(1-\frac{\operatorname{var} \mu}{\operatorname{var} q}\right) \rho+\frac{\operatorname{var} \mu}{\operatorname{var} q} \varphi
$$

Conclui-se, então, que o estimador de mínimos quadrados ordinário de $\rho$ é viciado para cima nas seguintes condições: ${ }^{11}$

$$
p \lim \hat{\rho}>\rho \text { se } \varphi>\rho
$$

A hipótese de que a autocorrelação entre as taxas de câmbio real de equilíbrio de longo prazo seja positiva é uma hipótese bastante plausível, e a condição de que esta correlação seja maior do que a autocorrelação do desvio da taxa de câmbio real observada em relação à taxa de equilíbrio de longo prazo é também uma hipótese bastante factível. Não é surpresa, portanto, que as estimativas do parâmetro $\rho$ na regressão (l) ou não rejeitem a hipótese de raiz unitária ou então obtenham coefi-

11 Quando $\operatorname{var}(\mu) \rightarrow 0$, ou quando $\operatorname{var}(q) \rightarrow \infty$, o estimador de $\rho$ é consistente. Por outro lado, se as variáveis $\mu_{t}$ e $q_{t}$ não forem estacionárias e $\operatorname{sim} I(1)$, o limite em probabilidade de $\hat{\rho}$ é indeterminado, pois as variâncias de $\mu_{t}$ e $q_{t}$ são de ordem $T$ e o coeficiente de correlação entre $\mu_{t}$ e $\mu_{t-1}$ é de ordem 1 . 
cientes de inércia bastante elevados, indicando uma meia vida de três a quatro anos, quando na realidade tal fato não ocorre porque, neste caso:

$$
\rho<p \lim \hat{\rho}<\varphi
$$

\section{PROCESSO ESTOCÁSTICO DA TAXA DE CÂMBIO REAL}

Os modelos apresentados na terceira seção mostram que a taxa de câmbio real de equilíbrio de longo prazo $(\mu)$ depende de um conjunto de variáveis $(x)$ de acordo com o modelo, que de um modo geral abrange preferências, tecnologia, política fiscal e sua composição, e a taxa de juros internacional. Analiticamente, a taxa de câmbio real de equilíbrio de longo prazo poderia ser especificada por uma equação do tipo:

$$
\mu_{t}=\beta(L)^{\prime} x_{t}+v_{t}
$$

onde $\beta(L)$ é um vetor de parâmetros com defasagens distribuídas e $v_{t}$ um termo aleatório que segue um processo autorregressivo. O problema desta especificação é que a taxa de câmbio real de equilíbrio de longo prazo não é uma variável observável e sua estimação depende de alguma hipótese que estabeleça uma ligação entre as taxas de curto e de longo prazo. A paridade do poder de compra será interpretada aqui como a hipótese de que a diferença entre a taxa de câmbio real de equilíbrio de curto prazo e a taxa de câmbio real de equilíbrio de longo prazo segue um processo estacionário, como indicado na equação (47).

A taxa de câmbio real de equilíbrio de longo prazo tem um processo estacionário quando o vetor $x_{t}$ for estacionário. Isto é:

$$
E \mu_{t}=\beta(1)^{\prime} E x_{t}=\beta(1)^{\prime} x=\mu
$$

onde $E x_{t}=x$. Admita-se que neste caso a taxa $\mu_{t}$ seja representada por um processo autorregressivo de primeira ordem,

$$
\mu_{t}=(1-\varphi) \mu+\varphi \mu_{t-1}+\vartheta_{t}
$$

onde $\phi$ é o coeficiente de autorregressão. Substituindo-se esta expressão em (42), obtém-se um processo ARMA $(2,1)$ para a taxa de câmbio real: 


$$
q_{t}=(1-\rho)(1-\varphi) \mu+(\rho+\varphi) q_{t-1}-\rho \varphi q_{t-2}+\xi_{t}-\theta \xi_{t-1}
$$

O parâmetro $\theta$ é o parâmetro da média móvel e $\xi_{t}$ é a variável aleatória com as propriedades tradicionais desta especificação. ${ }^{12}$

Quando o vetor $x_{t}$ não for estacionário, a taxa de câmbio real de equilíbrio de longo prazo também não será estacionária. Neste caso, cabe discutir três possibilidades. Na primeira, a esperança matemática do vetor $x_{t}$ é definida, mas varia ao longo do tempo, enquanto as covariâncias variam apenas com o intervalo de tempo. Na seção anterior mostrou-se que nesta situação o estimador do parâmetro $\rho$ da regressão $(\mathrm{l})$ é viciado para cima. Admita-se que a esperança matemática da taxa de câmbio real de longo prazo seja, então, dada por uma tendência determinística,

$$
E \mu_{t}=\mu+\gamma t
$$

e que esta taxa seja representada pelo seguinte processo autorregressivo de primeira ordem:

$$
\mu_{t}-E \mu_{t}=\varphi\left(\mu_{t-1}-E \mu_{t-1}\right)+\vartheta_{t}
$$

onde $\varphi$ é o coeficiente de autorregressão e $\vartheta$ o termo aleatório. Substituindo-se esta expressão em (42) chega-se, então, com um pouco de álgebra, ao seguinte processo estocástico para a taxa de câmbio real: ${ }^{13}$

$$
q_{t}=\kappa+(1-\rho)(1-\varphi) \gamma t+(\rho+\varphi) q_{t-1}-\rho \varphi q_{t-2}+\xi_{t}-\theta \xi_{t-1}
$$

Esta equação corresponde a um processo ARMA $(2,1)$, que inclui um termo determinístico, ou de modo equivalente: a taxa de câmbio real sem a tendência determinística segue um processo ARMA $(2,1)$. Obviamente, se $\gamma=0$ tem-se o caso anterior.

12 Quando $\mu_{t}$ for gerado por um processo ARMA $(1,1), q$ será um processo ARMA $(2,2)$.

13 Usando-se o operador de defasagem L, tem-se: $q_{t}=\mu_{t}+\frac{\varepsilon_{t}}{1-\rho L}$ e $\mu_{t}=E \mu_{t}+\frac{\vartheta_{t}}{1-\varphi L}$. Substituindo-se esta equação na anterior: $q_{t}=E \mu_{t}+\frac{\varepsilon_{t}}{1-\rho L}+\frac{\vartheta_{t}}{1-\varphi L}$. Logo, multiplicando-se ambos os lados desta expressão por $(1-\rho L)(1-\varphi L)$, obtém-se a equação $(61)$. A constante $\kappa$ é dada pela seguinte expressão: $\kappa=(1-\rho)[(1-\varphi) \mu+\varphi \gamma]+(1-\varphi) \rho \gamma$. 
Na segunda possibilidade, quando a taxa de câmbio real de longo prazo não é estacionária e todos os momentos dependem do tempo, o limite em probabilidade do estimador de $\rho$ é indeterminado. Admita-se que nesta hipótese a taxa de câmbio real de longo prazo tenha uma tendência estocástica, de acordo com um passeio aleatório com constante (drift). Isto é:

$$
\mu_{t}=\delta+\mu_{t-1}+\vartheta_{t}
$$

onde $\delta$ é a constante (drift) e $\vartheta$ um ruído branco. Combinando-se esta equação com a equação (4l) conclui-se que a taxa de câmbio real segue um processo não estacionário ARIMA $(1,1,1)$ :

$$
\Delta q_{t}=(1-\rho) \delta+\rho \Delta q_{t-1}+\xi_{t}-\theta \xi_{t-1}
$$

O parâmetro $\theta$, como nos demais casos, representa o parâmetro da média móvel.

As equações (58) e (63) são casos particulares da equação (61). Com efeito, no teste da hipótese nula $H_{0}: \varphi=1$, contra a hipótese alternativa $H_{1}: \varphi<1$, a não rejeição da hipótese nula implica uma raiz unitária para a taxa de câmbio real, como especificado em (63). No modelo da equação (61), reescrita aqui por conveniência, ${ }^{14}$

$$
q_{t}=a_{0}+a_{1} q_{t-1}+a_{2} q_{t-2}+a_{3} t+\xi_{t}-\theta \xi_{t-1}
$$

este teste corresponde ao teste da hipótese nula $H_{0}: a_{1}+a_{2}=1, a_{3}=0$, contra a hipótese alternativa $H_{1}: a_{1}+a_{2}<1, a_{3}>0$.

A rejeição da hipótese nula de que $\varphi=1$ descarta a possibilidade de raiz unitária, e neste caso pode-se testar a hipótese nula $H_{0}: \gamma=0$, de inexistência de tendência determinística para a taxa de câmbio real, contra a hipótese alternativa $H_{1}: \gamma>0$. Este teste é equivalente ao teste da hipótese nula $H_{0}: a_{3}=0$, contra a alternativa $H_{1}: a_{3}>0$ na equação (64). Caso esta hipótese nula não seja rejeitada, tem-se a especificação da equação (58).

Na terceira possibilidade, quando o vetor $x_{t}$ não for estacionário, admite-se que a esperança matemática da taxa de câmbio real de longo prazo varie ao longo do tempo. Supondo-se que a diferença entre a taxa de câmbio real de longo prazo e

14 Uma possibilidade que não deve deixar de ser considerada é de que a variância dos erros da regressão (64) siga um modelo GARCH porque os componentes da taxa de câmbio real têm um comportamento que pode ser descrito por este tipo de modelo. 
sua média seja o processo autorregressivo da equação (60), a taxa de câmbio real de curto prazo é dada por:

$$
q_{t}=E q_{t}+\frac{(1-\theta L)}{(1-\rho L)(1-\varphi L)} \xi_{t}
$$

Neste caso, a taxa de câmbio real de curto prazo segue um processo autorregressivo com média variável, igual à média da taxa de longo prazo, e o teste usual de raiz unitária não é aplicável.

\section{CONCLUSÃO}

$\mathrm{O}$ valor adicionado deste trabalho consiste em demonstrar duas proposições. A primeira é de que o quebra-cabeça da paridade do poder de compra resulta da hipótese mantida de supor que a taxa de câmbio real de equilíbrio de longo prazo é constante. Quando esta taxa varia com o tempo, como indica a teoria econômica, as estimativas do coeficiente de autorregressão da taxa de câmbio real são viciadas para cima. ${ }^{15}$

A segunda proposição é de que a rejeição da hipótese de que a taxa de câmbio real é estacionária não é equivalente à rejeição da hipótese da paridade do poder de compra, porque se a taxa de câmbio real não for estacionária ela pode ser cointegrada com a taxa de câmbio real de equilíbrio de longo prazo.

\section{REFERENCLAS}

BALASSA, Bela. The purchasing-power parity doctrine: a reappraisal. Journal of Political Economy, 72, p. 584-596, 1964.

CASSEL, Gustav. Abnormal deviations in international exchanges. Economic Journal, 28, p. 413-415, 1918.

DICKEY, David A.; FULLER, Wayne A. Likelihood ratio statistics for autoregressive time series with a unit root. Econometrica, 49, p. 1057-1072, 1981.

DORNBUSCH, Rudiger. Expectations and exchange rate dynamics. Journal of Political Economy, 84, p. 1161-1176, 1976.

EDWARDS, Sebastian. Real exchange rates, devaluation and adjustment. Cambridge: Ma.: MIT Press, 1989.

15 A análise apresentada neste trabalho sobre a taxa de câmbio real pode ser aplicada para outras variáveis macroeconômicas, cujos valores de equilíbrio de curto prazo sejam diferentes dos valores de equilíbrio de longo prazo. 
FLEMING, J. Marcus. Domestic financial policies under fixed and under floating exchange rates. IMF Staff Papers 9, p. 369-379, 1962.

HARROD, Roy. International economics. Cambridge: Cambridge University Press, 1939.

HINKLE, Lawrence E.; MONTIEL, Peter J. Exchange rate misalignment concepts and measurement for developing countries. Oxford: Oxford University Press, 1999.

MICHAEL, Panos; NOBAY, A. Robert; PEEL, David A. Transactions costs and nonlinear adjustment in real exchange rates: an empirical investigation. Journal of Political Economy, 105, p. 862-879, 1997.

MONTIEL, Peter. Determinants of the long-run equilibrium real exchange rate: an analytical model. In: HINKLE, Lawrence E.; MONTIEL, Peter J. (Org.), Exchange rate misalignment, concepts and measurement for developing countries. Oxford: Oxford University Press, 1999.

MUNDELL, Robert A. Capital mobility and stabilization policy under fixed and flexible exchange rates. Canadian Journal of Economics and Political Science, 29, p. $475-485,1963$.

OBSTFELD, Maurice; ROGOFF, Kenneth. Exchange rate dynamics redux. Journal of Political Economy, 103, p. 624-660, 1995.

. Foundations of international economics. Cambridge: MA: MIT Press, 1996.

ROGOFF, Kenneth. The purchasing power parity puzzle. Journal of Economic Literature, 34, p. 647-668, 1996.

SALTER, Walter E. Internal and external balance: the role of price and expenditure effects. Economic Record, 35, p.226-238 1959.

SAMUELSON, Paul A. Theoretical notes on trade problems. Review of Economics and Statistics, 46, p. 145-154, 1964.

SWAN, Trevor W. Economic control in a dependent economy. Economic Record, 36, p. 51-66, 1960.

TAYLOR, Mark P.; PEEL, David A.; SARNO, Lucio. Nonlinear mean-reversion in real exchange rates: toward a solution to the purchasing power parity puzzles. International Economic Review, 42, p. 1015-1042, 2001.

WILLIAMSON, John. Estimating equilibrium exchange rates. Washington, D.C.: Institute for International Economics, 1974. 